\title{
Earlier Treatment of Vulvovaginal Atrophy in Post-Menopausal Women May Improve Treatment Outcomes
}

\author{
Palacios $\mathrm{S}^{1 *}$, Panay $\mathrm{N}^{2}$, Sánchez-Borrego $\mathbf{R}^{3}$, Particco $\mathbf{M}^{4}$ and Djumaeva $\mathrm{S}^{4}$ \\ ${ }^{1}$ Centro Medico Instituto Palacios Salud de la Mujer, Spain \\ ${ }^{2}$ Queen Charlotte's and Chelsea \& Westminster Hospitals, Imperial College, UK \\ ${ }^{3}$ DIATROS, Clínica de Atención a la Mujer, Spain \\ ${ }^{4}$ Shionogi Europe, $U K$
}

Submission: July 13, 2019 , Published: July 26, 2019

*Corresponding author: Santiago Palacios, Centro Medico Instituto Palacios Salud de la Mujer, Spain

Abstract

Objective: To determine whether early ospemifene treatment, when vulvovaginal atrophy (VVA) symptoms are still moderate rather than severe, leads to greater treatment benefits.

Methods: This post-hoc analysis used the clinical trial data from the two ospemifene pivotal 12-week Phase III trials, which enrolled women with at least one moderate or severe symptom of vaginal dryness or dyspareunia. The participants were split into those with a moderate most bothersome symptom (MBS) and those with a severe MBS, as reported at baseline. The impact of ospemifene treatment on improvement and relief of their MBS was evaluated. The impact of treatment on all VVA symptoms, by baseline severity, was also recorded.

Results: In the first efficacy study, a greater proportion of women with MBS of dyspareunia and vulvar/vaginal itching/irritation at baseline improved if they had moderate rather than severe symptoms. In the second efficacy study, the opposite was observed. When all moderate/severe symptoms at baseline were included in the analysis, there was no difference in improvement based on severity. However, when all symptoms were analysed, more women experienced relief if treatment was started when symptoms were moderate $(75 \%)$ rather than severe (58\%).

Conclusion: Ospemifene improves all moderate or severe VVA symptoms, regardless of baseline severity, but earlier treatment of VVA symptoms, when symptoms are moderate rather than severe, may be associated with greater treatment benefits, as indicated by the higher 'cure' rate in women with moderate VVA symptoms at baseline.

Keywords: Post-menopausal, Genitourinary syndrome, Vulvar and vaginal atrophy, VVA, Vaginal dryness, Dyspareunia, Selective estrogen receptor modulator, Ospemifene

Abbreviations: HCP: Healthcare Professional, MBS: Most Bothersome Symptom, SERM: Selective Estrogen Receptor Modulator, QoL: Quality of Life, VVA: Vulvovaginal Atrophy

\section{Introduction}

Vulvovaginal atrophy (VVA) is a component of the genitourinary syndrome of menopause [1] and is associated with decreased estrogenization of the vaginal tissue [2]. Symptoms include vaginal dryness, dyspareunia (vaginal pain associated with sexual activity), vaginal and/or vulvar irritation or itching, dysuria, and vaginal bleeding associated with sexual activity $[1,3]$. Women with VVA rarely experience only one symptom, with the European Vulvovaginal Epidemiological Survey (EVES) recently reporting an average of five symptoms (range 1-14) [4].
Approximately $50-60 \%$ of post-menopausal women experience VVA symptoms [1] and these can adversely impact different aspects of well-being [4,5], such as the quality of sex lives and relationships, self-esteem, and everyday activities [6-8]. Recently, the EVES found that VVA symptoms and their severity correlated strongly with patient quality of life (QoL) [4,9]. Specifically, QoL was significantly lower in women with severe symptoms versus those without severe symptoms in all women, with women with severe urinary symptoms showing the greatest reduction 
[9]. Decrements in QoL were particularly evident for emotional well-being, self-concept/body image, and mobility [9]. Importantly, reductions in QoL in women with VVA have been reported to be comparable with those found in serious conditions, such as arthritis, chronic obstructive pulmonary disease, asthma, and irritable bowel syndrome [2].

Unlike hot flushes and night sweats, vaginal atrophic changes do not resolve spontaneously with time, are often progressive, and frequently require treatment [10]. However, some women regard VVA symptoms as manifestations of the natural ageing process and do not seek help [1]. In the Vaginal Health: Insights, Views \& Attitudes (VIVA) survey, $42 \%$ of post-menopausal women were able to relate VVA symptoms to the menopause, but only $4 \%$ of women surveyed attributed their vaginal symptoms to vaginal atrophy [11].

Despite its high prevalence and negative impact on QoL, VVA is not only under-reported by women, but also under-diagnosed and under-treated by healthcare professionals (HCPs) [1]. The European REal women's VIew of treatment options for menopausal Vulvar/Vaginal changEs (REVIVE-EU) survey found that $60 \%$ of women on treatment had discussed VVA with their HCP, but only $10 \%$ of HCPs had initiated the discussion about VVA symptoms with their patients [12].

The combination of under-reporting, under-diagnosis, and under-treatment may lead to many women presenting with severe VVA symptoms, at an advanced stage of disease. Potentially, this has important consequences, as not only are the symptoms unlikely to improve spontaneously, but the anatomical changes of atrophy will not improve without treatment. Such changes may, sometimes rapidly, lead to vaginal stenosis and more severe consequences, including vaginal occlusion [13].

Also, delaying treatment of vaginal atrophy may lead to vaginal and urinary tract infections (UTIs); increasing vaginal atrophy is associated with increasing overactive bladder symptoms, recurrent UTIs and non-infective cystitis, as well as bacterial vaginosis and thrush infections [14]. Ospemifene, a selective estrogen receptor modulator (SERM), is a novel oral treatment for moderate to severe symptomatic VVA in post-menopausal wom- en who are not candidates for local vaginal oestrogen therapy [15]. Ospemifene is the only SERM which was developed specifically for VVA [16]. Unlike other SERMs, ospemifene has an oestrogenic effect on the vaginal epithelium [17]; after 12 weeks of treatment, an increase from baseline in the percentage of superficial cells has been observed, coupled with a reduction in parabasal cells and vaginal $\mathrm{pH}$ [18-20]. Moreover, the improvements in vaginal physiology were accompanied by consistent improvements in the most bothersome symptom (MBS) of vaginal dryness $[18,20]$ or dyspareunia $[18,19]$.

Whilst the need for treatment is clearly demonstrated by the anatomical changes that could be mitigated, it would be of interest to determine whether early treatment also leads to better symptom alleviation. Hence, the aim of this post-hoc analysis was to assess whether early treatment of VVA symptoms, when they are still moderate rather than severe, leads to greater treatment benefits.

\section{Methods}

Using the clinical trial data from the two ospemifene pivotal 12-week Phase III trials [18-20], which enrolled women with at least one moderate or severe symptom of vaginal dryness or dyspareunia, the participants were split into two groups: those with a moderate MBS and those with a severe MBS, as reported at baseline (symptom severity was reported by the patient at baseline as 'none', 'mild', 'moderate', or 'severe').

The analysis evaluated the impact of 12 weeks of ospemifene treatment on: improvement (reflecting the percentage of patients who achieved a reduction in severity score of one or more steps [on a 3-step scale], which included patients whose baseline score changed from 'severe' to 'none', 'mild', or 'moderate'; from 'moderate' to 'mild' or 'none'; and from 'mild' to 'none'); relief (reflecting the percentage of patients who had a severity score of 'mild' or 'none' at 12 weeks). In addition, the impact of treatment on all VVA symptoms, by baseline severity, was recorded for all women. This descriptive analysis did not include any formal statistical testing and hence the results are presented as numerical findings.

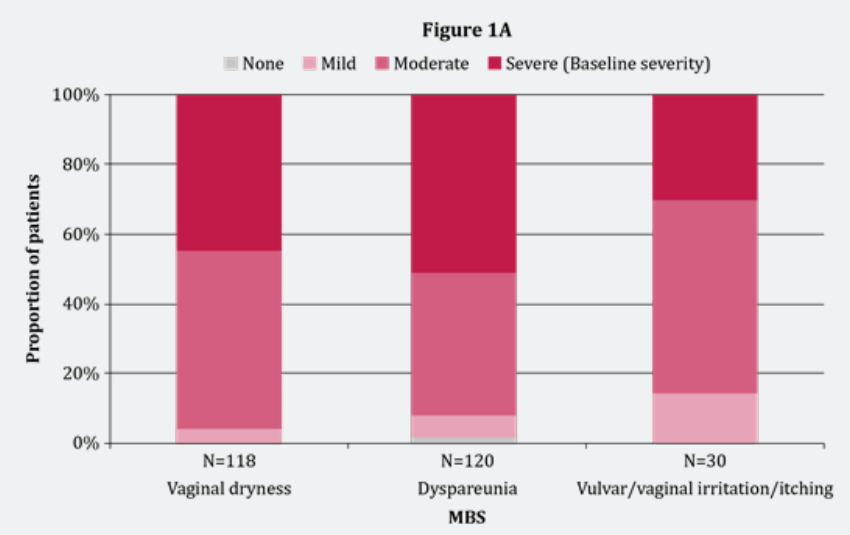

Figure 1A: Baseline severity distribution of the most bothersome symptom (MBS, study 15-50310). 


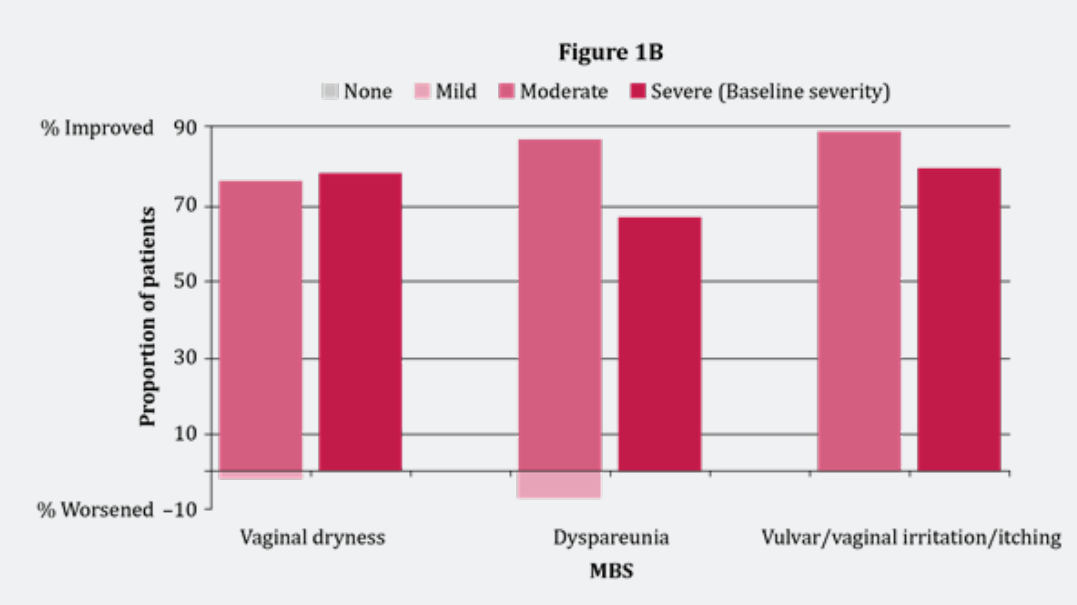

Figure 1B: Impact of baseline severity on improvement after 12 weeks of ospemifene treatment (most bothersome symptom [MBS], study 15-50310).

\section{Results}

In the first pivotal efficacy study (15-50310, NCT00276094) [18], all women who selected a VVA symptom as their MBS were enrolled. There were very few women who selected dysuria or post-coital bleeding, so those groups were too small to include in the analysis. In this study, $46 \%$ of women selected dyspareunia as the MBS, $39 \%$ vaginal dryness, and $13 \%$ vulvar/vaginal irritation/itching [18]. The baseline severity distribution of the MBS in the first pivotal efficacy study (15-50310) is presented in Figure 1A. The impact of 12 weeks of ospemifene treatment on improvement, by baseline severity, can be seen in Figure 1B; more women with moderate compared with severe MBS of dyspareunia $(86.2 \%$ versus $65.5 \%)$ or vulvar/vaginal irritation/ itching $(88.2 \%$ versus $77.8 \%)$ showed improvement with ospemifene, although the differences were small (no difference for vaginal dryness).
The baseline severity distribution in the second pivotal efficacy study (15-50821, NCT00729469), where only women with an MBS of vaginal dryness [20] or dyspareunia [19] were enrolled, can be seen in Figure 2A. In this study, the improvement after 12 weeks of ospemifene treatment was slightly better in the severe population compared with the population whose MBS was moderate at baseline (vaginal dryness: $76.8 \%$ versus $64.1 \%$, dyspareunia: $81.1 \%$ versus $77.5 \%$ ); however, the differences were again small (Figure 2B).The baseline severity distribution of all VVA symptoms is shown in Figure 3A. Comparing the impact of 12 weeks of ospemifene treatment on improvement of all moderate versus all severe symptoms at baseline, revealed only a small difference between the two populations: $75 \%$ versus $79 \%$ (Figure 3B).

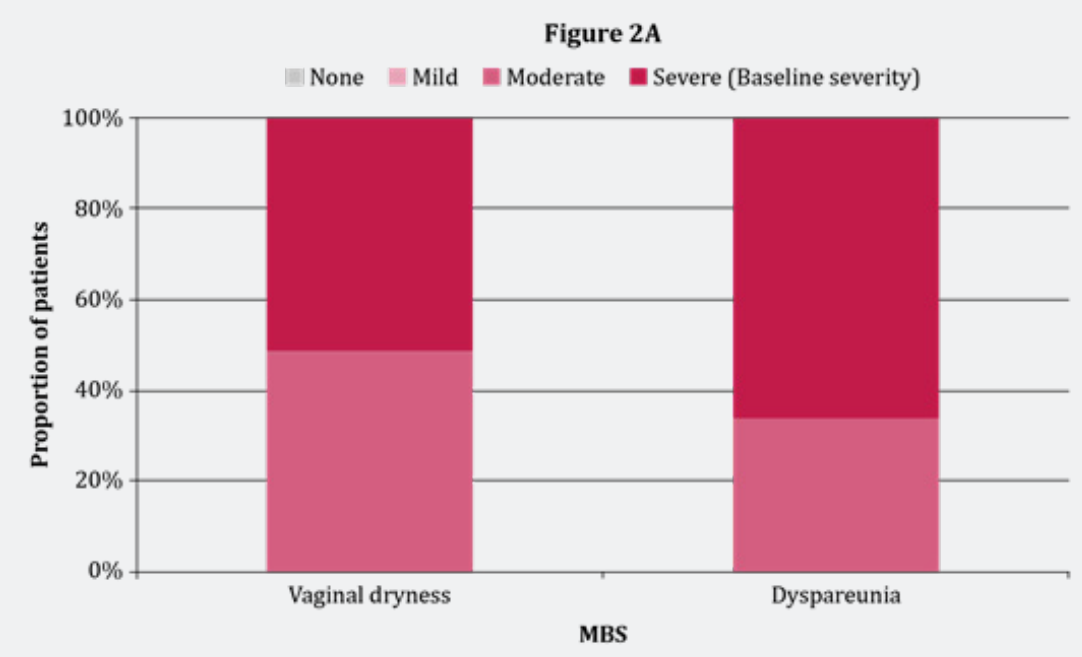

Figure 2A: Baseline severity distribution of the most bothersome symptom (MBS, study 15-50821). 


\section{Journal of Gynecology and Women's Health}

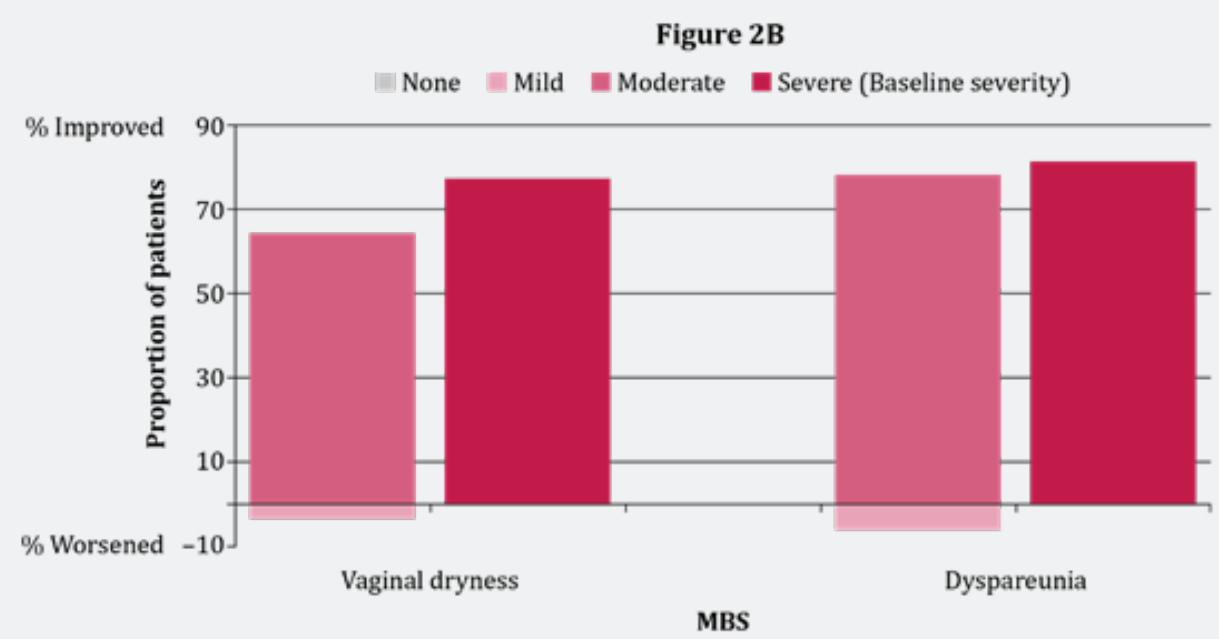

Figure 2B: Impact of baseline severity on improvement after 12 weeks of ospemifene treatment (most bothersome symptom [MBS], study 15-50821).

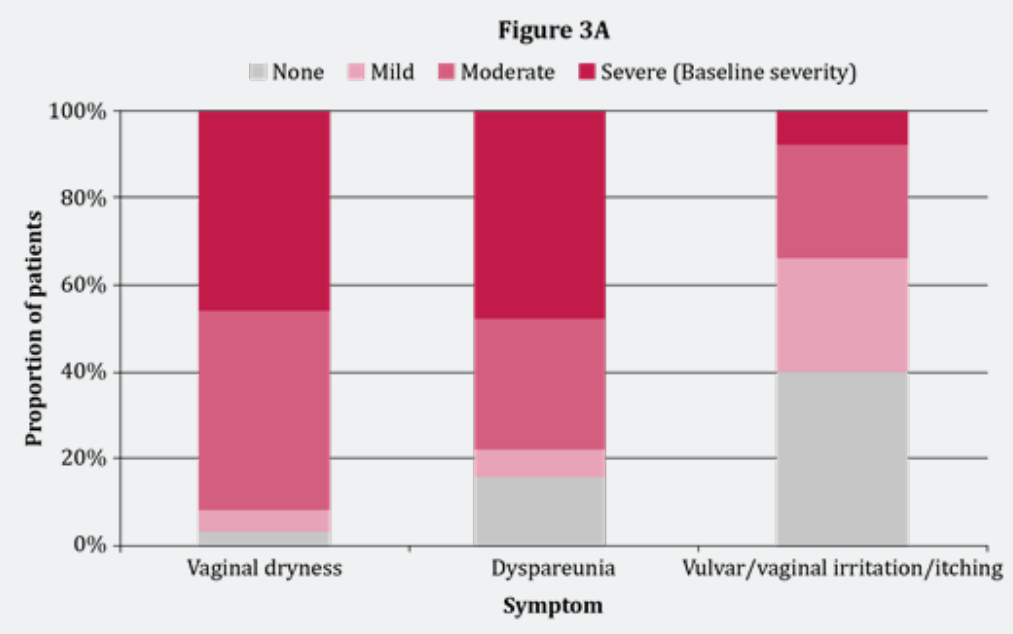

Figure 3A: Baseline severity distribution of all VVA symptoms (studies 15-50821 and 15-50310 combined).

Figure 3B

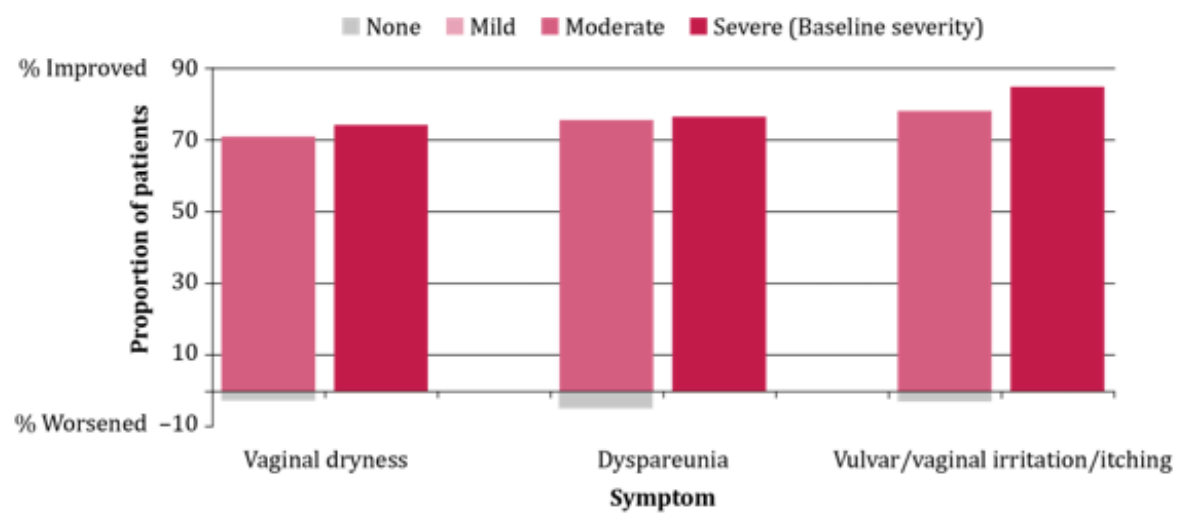

Figure 3B: Impact of baseline severity on improvement of all moderate or severe VVA symptoms after 12 weeks of treatment (studies 1550821 and 15-50310 combined). 


\section{Journal of Gynecology and Women's Health}

When we evaluated the impact of 12 weeks of ospemifene treatment on relief (or 'cure'), by baseline severity, the proportion of women who experienced relief after treatment was higher in those women who had moderate MBS at baseline compared with those who had severe MBS and this effect was evident in both pivotal studies (Figure $4 \mathrm{~A} \& \mathrm{~B}$ ). A comparison of the impact of 12 weeks of ospemifene treatment on all moderate or severe symptoms, by baseline severity, also confirmed that the proportion of patients with symptoms that were only mild or non-existent after treatment was higher in patients with symptoms that were moderate at baseline compared with those whose symptoms were severe at baseline; the 'cure' rate in women with moderate symptoms was around $75 \%$, whereas it was $58 \%$ in women with severe symptoms (Figure 5).

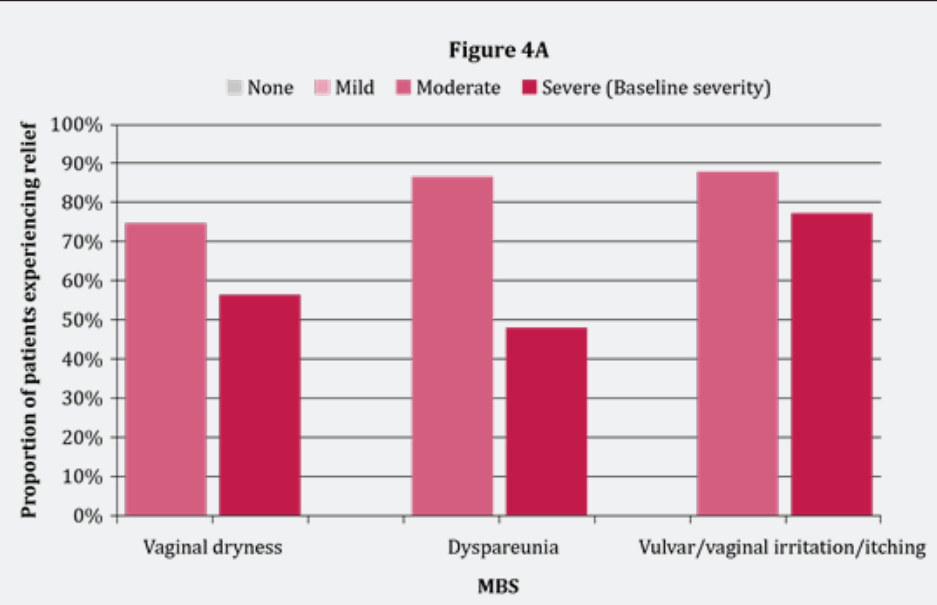

Figure 4A: Impact of baseline severity on relief after 12 weeks of ospemifene treatment (most bothersome symptom [MBS], study 1550310).

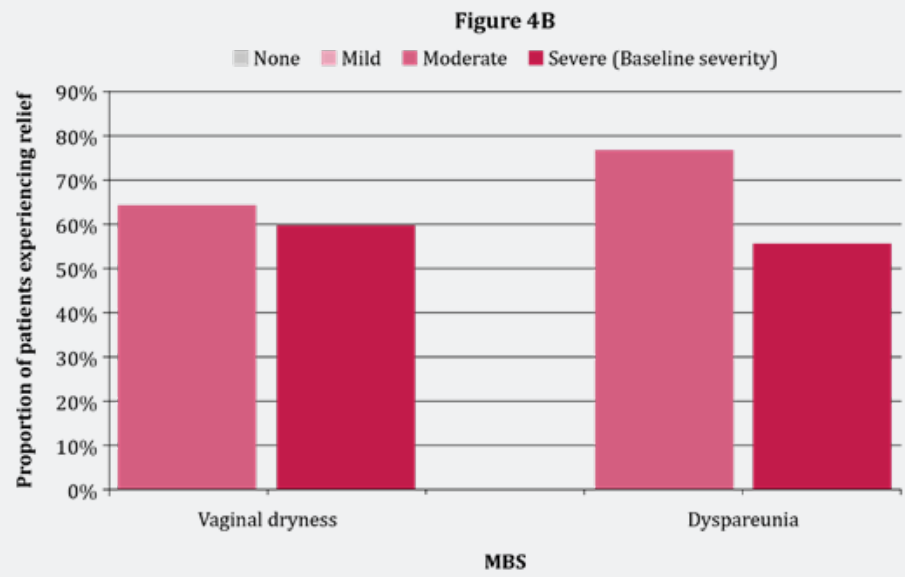

Figure 4B: Impact of baseline severity on relief after 12 weeks of ospemifene treatment (most bothersome symptom [MBS], study 1550821).

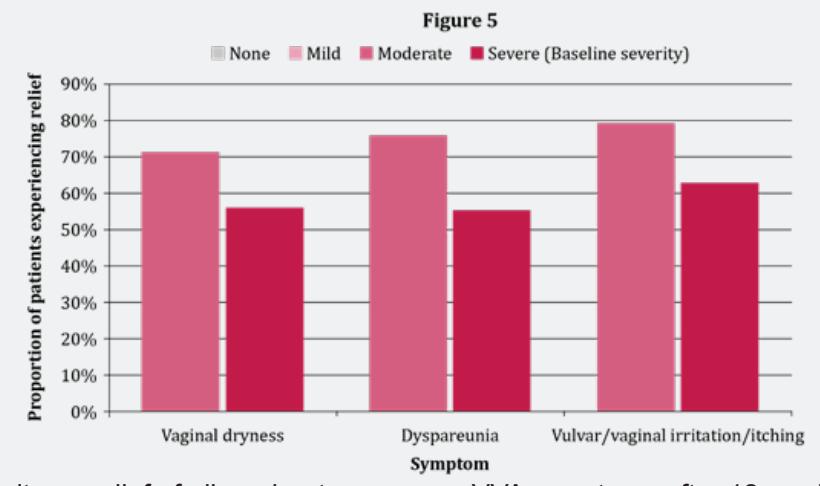

Figure 5: Impact of baseline severity on relief of all moderate or severe VVA symptoms after 12 weeks of treatment (studies 15-50821 and 15-50310 combined). 


\section{Discussion}

The current post-hoc analysis showed that in the first ospemifene efficacy study, more women showed greater improvement with moderate MBS of dyspareunia and vulvar/vaginal itching/irritation at baseline than did women whose MBS was severe. In the second efficacy study, this was the other way around (i.e. more women improved in the group with severe MBS at baseline).

Because $79.5 \%$ of the women enrolled in the pivotal studies combined had more than one moderate or severe symptom of VVA (two symptoms: 47.3\%; three symptoms: $23.5 \%$; four symptoms: $7.1 \%$; five symptoms: $1.6 \%$ ] [21], selecting one MBS per patient would underestimate the impact of treatment. Therefore, we also considered the effect of early treatment based on all symptoms of VVA. When we compared all moderate or severe symptoms at baseline, there was no impact on improvement based on severity (there was only $4 \%$ difference in improvement of all moderate or severe symptoms). Taken together, these results suggest that ospemifene improves all moderate or severe VVA symptoms, regardless of baseline severity.

This is a reassuring finding, as there is no reason to restrict treatment with ospemifene to those women with the most severe symptoms: ospemifene is equally beneficial for women with moderate symptoms. However, our analysis of all symptoms revealed that more women experienced relief of their symptoms if those were treated when moderate rather than severe $(17 \%$ difference in relief of all moderate or severe symptoms). These findings suggest that earlier treatment of VVA symptoms, when symptoms are moderate rather than severe, may be associated with greater treatment benefits; both because of the higher 'cure' rate and because it prevents the sequelae of ongoing atrophy, which may lead to vaginal stenosis, or even occlusion [13].

Indeed, the International Menopause Society (IMS) recommends that VVA treatment is started early, before irrevocable atrophic changes have occurred, and that it is continued to maintain the benefits [22]. The EVES also concluded that treatment of VVA should be initiated early, before the appearance of significant anatomical changes and despite the absence of obvious VVA signs; the authors stressed that objective gynaecological assessment of VVA was important to help rule out conditions with VVA-type symptoms and for early treatment initiation [4]. The lower QoL in women with severe VVA symptoms that was observed in the EVES may reflect a delay in visiting their doctor, compromising the management of VVA [4]. Most recently, the EVES data were used to evaluate the association between treatments for VVA and symptom frequency and severity, QoL, and sexual functioning in post-menopausal women [23]. Women on VVA treatment presented with more severe symptoms, which suggests that treatment tends not to be initiated when symptoms commence, but when they have become more severe and are causing considerable distress [23]. These findings lead to the same conclusion that the treatment of VVA should ideally be initiated at early stages of the disease, to prevent irreversible changes [23].

Interestingly, a recent study, AN evaluation of GEnitourinary symptoms in perimenopausaL women (ANGEL), has shown that VVA and its symptoms are common across the menopausal transition, with the prevalence ranging from $19.2 \%$ among women aged $40-45$ years to $53.8 \%$ in women aged $52-55$ years; hence, early identification of this condition may favour early therapeutic intervention [24]. Direct questioning by HCPs during history taking in peri- and post-menopausal women has been highlighted as an important aspect of VVA diagnosis and management $[5,14]$. Also, because most women are not familiar with the term 'vaginal atrophy' [11], initiating discussion about the range of possible VVA symptoms may be more useful [14].

Limitations of the present research include the post-hoc nature of the analysis, the lack of formal statistical testing, and the lack of data on the length of time that the women had been suffering with moderate or severe VVA symptoms. Additionally, by not undertaking a prospective analysis, we were more likely to show better results for moderate VVA symptoms, as these would be more easily 'cured' than severe VVA symptoms, because of the lower scores at baseline.

An important strength is the fact that this analysis was based on a large population of women with VVA who had been evaluated in the controlled environment of double-blind randomized controlled trials. Also, this analysis should raise awareness of VVA as a chronic condition with a progressive pathology. Thus, like other chronic conditions, VVA should be promptly diagnosed and treated.

\section{Conclusion}

Despite methodological limitations, the present findings suggest that ospemifene improves all moderate or severe VVA symptoms, regardless of baseline severity; however, earlier treatment of VVA symptoms, when symptoms are moderate rather than severe, may be associated with greater treatment benefits, as indicated by the higher 'cure' rate in women with moderate VVA symptoms at baseline.

\section{Acknowledgements}

The authors were assisted in the preparation of the manuscript by Solaris Health, who provided writing and editorial support, funded by Shionogi Europe.

\section{Conflicts of Interest}

SP has received honoraria or consulting fees from Pfizer, Amgen, MSD, Gynea, Procare Health, Bayer, Lacer, Serelys and Shionogi Europe and has participated in company-sponsored speakers' bureaus for Pfizer, Abbott, Bioiberica, Shionogi Europe, Amgen, Novo Nordisk, Teva, Bayer Healthcare, Serelys, Exeltis and Gedeon Richter. NP has received honoraria for lecturing 
and acting in an advisory capacity for several pharmaceutical companies, including Abbott, Bayer, Besins, Mithra, MSD, Mylan, Novo Nordisk, Pfizer, Se-cure and Shionogi Ltd. RSB has received research funding from Bayer and Procare Health and honoraria from Shionogi Europe, Lacer, Mylan, Theramex and Seid Pharmaceuticals. MP and SD are employees of Shionogi Europe.

\section{References}

1. Naumova I, Castelo-Branco C (2018) Current treatment options for postmenopausal vaginal atrophy. Int J Women's Health 10: 387-395.

2. DiBonaventura M, Luo X, Moffatt M, Bushmakin AG, Kumar M, et al. (2015) The association between vulvovaginal atrophy symptoms and quality of life among postmenopausal women in the United States and Western Europe. J Women's Health 24(9): 713-722.

3. Kingsberg S, Larkin L, Krychman M, Parish SJ, Bernick B, et al. (2019) WISDOM survey: attitudes and behaviors of physicians toward vulvar and vaginal atrophy (VVA) treatment in women including those with breast cancer history. Menopause 26(2): 124-131.

4. Palacios S, Nappi RE, Bruyniks N, Particco M, Panay N, et al. (2018) The European Vulvovaginal Epidemiological Survey (EVES): prevalence, symptoms and impact of vulvovaginal atrophy of menopause. Climacteric 21(3): 286-291.

5. Belardo A, Pilnik S, Gelin M, Garcia P (2018) Genito Urinary Syndrome of Menopause "a new terminology for an ever-present symptomatology". J Repro \& Sexual Disord 1(5): 138-145.

6. Palacios S, Cancelo MJ, Castelo BC, Llaneza P, Molero F, et al. (2017) Vulvar and vaginal atrophy as viewed by the Spanish REVIVE participants: symptoms, management and treatment perceptions. Climacteric 20(1): 55-61.

7. Nappi R, Mattsson L-A, Lachowsky M, Maamari R, Giraldi A (2013) The CLOSER survey: Impact of postmenopausal vaginal discomfort on relationships between women and their partners in Northern and Southern Europe. Maturitas 75(4): 373-379.

8. Nappi R, Palacios S, Panay N, Particco M, Krychman ML (2016) Vulvar and vaginal atrophy in four European countries: evidence from the European REVIVE Survey. Climacteric 19(2): 188-197.

9. Nappi RE, Palacios S, Bruyniks N, Particco M, Panay N, et al. (2019) The burden of vulvovaginal atrophy on women's daily living: implications on quality of life from a face-to-face real-life survey. Menopause 26(5): 485-491.

10. Sturdee DW, Panay N, International Menopause Society Writing Group (2010) Recommendations for the management of postmenopausal vaginal atrophy. Climacteric 13(6): 509-522.

11. Nappi R, Kokot-Kierepa M (2012) Vaginal Health: Insights, Views \& Attitudes (VIVA) - results from an international survey. Climacteric 15(1): 36-44.
12. Nappi R, Palacios S, Panay N, Rabe T, Krychman M, et al. (2015) Results of the European REVIVE (REal Women's VIew of Treatment Options for Menopausal Vulvar/Vaginal ChangEs) survey. Maturitas 81: 183.

13. Freedman MA (2008) Vaginal pH, estrogen and genital atrophy. Menopause Management. https://www.menopausemgmt.com/ vaginal-ph-estrogen-and-genital-atrophy/ (accessed April 2019).

14. Domoney C (2014) Treatment of vaginal atrophy. Women's Health 10(2): 191-200.

15. Nappi RE, Murina F, Perrone G, Villa P, Biglia N, et al. (2017) Clinical profile of women with vulvar and vaginal atrophy who are not candidates for local vaginal estrogen therapy. Minerva Ginecol 69(4): 370-380.

16. Rabe T, Bruyniks N, Merkle E, Hadji P, Kuhl H, et al. (2015) Selective estrogen receptor modulators - an update (translated version)-joint statement by the German Society for Gynecological Endocrinology and Reproductive Medicine (DGGEF) and the German Professional Association of Gynecologists (BVF). J Reproduktionsmed Endokrinol 12(4): 287-317.

17. Palacios S, Cancelo MJ (2016) Clinical update on the use of ospemifene in the treatment of severe symptomatic vulvar and vaginal atrophy. Int J Women's Health 8: 617-626.

18. Bachmann GA, Komi JO, Ospemifene Study Group (2010) Ospemifene effectively treats vulvovaginal atrophy in postmenopausal women: results from a pivotal phase 3 study. Menopause 17(3): 480-486.

19. Portman DJ, Bachmann GA, Simon JA, Ospemifene Study Group (2013) Ospemifene, a novel selective estrogen receptor modulator for treating dyspareunia associated with postmenopausal vulvar and vaginal atrophy. Menopause 20(6): 623-630.

20. Portman D, Palacios S, Nappi RE, Mueck AO (2014) Ospemifene, a nonoestrogen selective oestrogen receptor modulator for the treatment of vaginal dryness associated with postmenopausal vulvar and vaginal atrophy: a randomised, placebo-controlled, phase III trial. Maturitas 78(2): 91-98.

21. Bruyniks N, Nappi RE, Castelo-Branco C, Villiers DTJ, Simon J (2016) Effect of ospemifene on moderate or severe symptoms of vulvar and vaginal atrophy. Climacteric 19(1): 60-65.

22. Baber RJ, Panay N, Fenton A, IMS Writing Group (2016) IMS Recommendations on women's midlife health and menopause hormone therapy. Climacteric 19(2): 109-150.

23. Panay N, Palacios S, Bruyniks N, Particco M, Nappi RE, et al. (2019) Symptom severity and quality of life in the management of vulvovaginal atrophy in postmenopausal women. Maturitas 124: 55-61.

24. Cagnacci A, Xholli A, Sclauzero M, Venier M, Palma F, et al. (2019) Vaginal atrophy across the menopausal age: results from the ANGEL study. Climacteric 22(1): 85-89. 
(C) This work is licensed under Creative
Your next submission with Juniper Publishers will reach you the below assets

- Quality Editorial service

- Swift Peer Review

- Reprints availability

- E-prints Service

- Manuscript Podcast for convenient understanding

- Global attainment for your research

- Manuscript accessibility in different formats ( Pdf, E-pub, Full Tsext, Audio)

- Unceasing customer service

Track the below URL for one-step submission https://juniperpublishers.com/online-submission.php 